\title{
Angle-Dependent Atomic Force Microscopy Single-Chain Pulling of Adsorbed Macromolecules from Planar Surfaces Unveils the Signature of an Adsorption-Desorption Transition
}

\author{
Lucie Grebíková, ${ }^{\dagger}$ Stuart G. Whittington, ${ }^{\ddagger}$ and Julius G. Vancso**i() \\ ${ }^{\dagger}$ Materials Science and Technology of Polymers, MESA+, Institute of Nanotechnology, University of Twente, P.O. Box 217, $7500 \mathrm{AE}$ \\ Enschede, The Netherlands \\ ${ }^{*}$ Department of Chemistry, University of Toronto, 80 St. George Street, Toronto, Ontario M5S 3H6, Canada
}

Supporting Information

ABSTRACT: The adsorption-desorption behavior of polymer chains is at the heart of macromolecular surface science and technology. With the current developments in atomic force microscopy (AFM), it has now become possible to address the desorption problem from the perspective of a single macromolecule. Here, we report on desorption of single polymer chains on planar surfaces by AFM-based single molecule force spectroscopy (SMFS) as a function of the pulling angle with respect to the surface-normal direction. SMFS experiments were performed in water with various substrates using different polymers covalently attached to the AFM probe tip. End-grafting at the AFM tip was achieved by surface-initiated polymerization using initiator functionalized tips. We found that the desorption force increases with a decreasing pulling angle, i.e., an enhanced adhesion of the polymer chain was observed. The magnitude of the desorption force shows a weak angular dependence at pulling angles close to the surface normal. A significant increase of the force is observed at shallower pulling from a certain pulling angle. This behavior carries the signature of an adsorption-desorption transition. The angular dependence of the normalized desorption force exhibits a universal behavior. We compared and interpreted our results using theoretical predictions for single-chain adsorption-desorption transitions.

\section{INTRODUCTION}

Polymer adhesion is a complex phenomenon that has high relevance in theoretical and experimental areas of research. Our ability to delineate the properties that determine polymer adhesion at the molecular level is crucial for the further development of innovative designs of surgical adhesives ${ }^{1}$ and polymer coatings with antifouling properties, ${ }^{2-4}$ biosensors, ${ }^{5,6}$ low-friction low-wear surfaces, ${ }^{7}$ and polymer-based technological glues. ${ }^{1,8}$

Macromolecular adhesion and desorption have been studied traditionally at the ensemble level of thermodynamics. These studies average over large populations of polymers. Therefore, individual characteristics of single-chain adsorption are hidden. With the current developments in atomic force microscopy (AFM) single molecule force spectroscopy (SMFS) techniques, it has now become possible not only to study polymer adhesion at the true molecular level but also to tackle directional dependence of the desorption problem (i.e., failure of physical adhesion). It has hitherto remained a grand challenge to address the adsorption/desorption of single polymer chains as a function of the pulling angle with respect to the plane of adsorption. Polymer adsorption-desorption has been investigated at the substrate surface-normal direction while the surface adsorbed chains were either picked up and stretched by the tip probe or firmly attached to the tip. In both cases, the force exerted on the cantilever was measured as a function of the distance from the surface. ${ }^{9-14}$ With the advances in the new generation of AFM instruments, it is now possible to control the tip position and direction with high precision with respect to the surface in three spatial directions, utilizing independent feedback control in the three Cartesian directions. The adsorbed polymer can then be pulled at different positions along the backbone (for this experiment, previous single-chain imaging is required) or also from the surface in different directions. However, modifications to the software of commercial instrumentation are still required for the latter experiment. To date, few studies have made use of this option. In our approach, ${ }^{15}$ the AFM tip is retracted perpendicularly to the surface with a constant velocity while the substrate is simultaneously moved in $x$ and $y$ directions. With precise control of the velocities, the desired pulling angle can be achieved. Kühner et al. ${ }^{16}$ performed experiments with polymers pinned to the AFM tip where the cantilever was retracted to a

Received: March 14, 2018

Published: May 1, 2018 
certain distance away from the surface, then moved parallel to the surface, with numerous repetitions of these steps, and so forth. A similar approach was employed in stretching one single DNA duplex repeatedly at different horizontal locations by controlling the motion of the XYZ stage under the AFM tip. ${ }^{17}$ These studies focused on force-extension measurements of individual polymers by SMFS in order to derive parameters relevant to polymer adhesion, conformation and mechanics.

Several theoretical and computational studies ${ }^{18-22}$ have addressed the polymer desorption from a substrate by varying the angle of pulling forces with respect to the substrate surface normal, in the range from substrate perpendicular to parallel pull. These models predict that, depending on the angle, the polymer favors either adsorption or desorption. To date, only some studies attempted to vary force directions and pull polymer chains laterally over the substrate to enhance polymer adsorption or to study single-polymer friction. 7,16,23,24 Thus, designed experiments to probe how the polymer behaves in the intermediate region remain essentially unexplored. Our goal in this study is to gain a microscopic understanding of the hitherto experimentally unaddressed adsorption-desorption transitions, i.e., how a polymer responds to an external force applied at various angles.

A theoretical model for pulling an adsorbed polymer at an angle has been considered by Orlandini and Whittington ${ }^{19}$ and by Osborn and Prellberg. ${ }^{21}$ Although the theoretical approaches used in the two papers are different, the underlying model and the results are identical. The model used is a partially directed walk on the simple cubic lattice. Consider the simple cubic lattice and attach the obvious coordinate system $(x, y, z)$, so that each vertex of the lattice has integer coordinates. A partially directed walk can take steps in the positive $x$-direction, in the positive $y$-direction, and in both the positive and negative $z$-directions, subjected to the walk not revisiting any already visited vertices of the lattice. The walk starts at the origin and is confined to the half-space $z \geq 0$, so that the plane $z=0$ represents an impenetrable surface at which adsorption can occur. The walk receives weights for each edge in $z=0$ and is subjected to force at the vertex of degree 1 that is not fixed at the origin. The model can be solved completely using generating function techniques and the thermodynamics (in the infinite walk limit) can be extracted from the singularity structure of the generating function. The principle result is the angular dependence of the critical force required for desorption as a function of a parameter, $T$, that plays the role of a reduced temperature.

In this paper, we chose two polymer systems that differ in hydrophobicity and their conformational states in water (used as medium), namely, poly(2-hydroxyethyl methacrylate) (PHEMA) and poly(methyl methacrylate) (PMMA). Detailed pulling angle dependence of the adsorption was studied for PHEMA on various substrates including mica, silica and highly ordered pyrolytic graphite (HOPG). PHEMA and PMMA were synthesized and end-grafted from an AFM tip via surfaceinitiated, quasi-living atom transfer radical polymerization (SIATRP). ${ }^{25}$ This approach allowed us to obtain end-grafted chains fastened to the tip with controlled chain length.

\section{RESULTS AND DISCUSSION}

The adhesion of PHEMA and PMMA end-grafted from the AFM tip was investigated by SMFS in aqueous solution on three different substrates, namely, mica, silica, and HOPG. PHEMA and PMMA were grown from initiator functionalized
AFM tips by ATRP with low enough grafting density to ensure the presence of individual polymer chains at relatively large distances, thus facilitating single-chain contact with the substrate. In the first series of experiments, the polymer chain was desorbed by pulling the polymer decorated AFM tip away perpendicularly from the surface. Subsequently, the angledependent single polymer chain adhesion experiments were carried out where the force response of the polymer chain was measured while it was pulled from the surface along different directions (Figure 1).

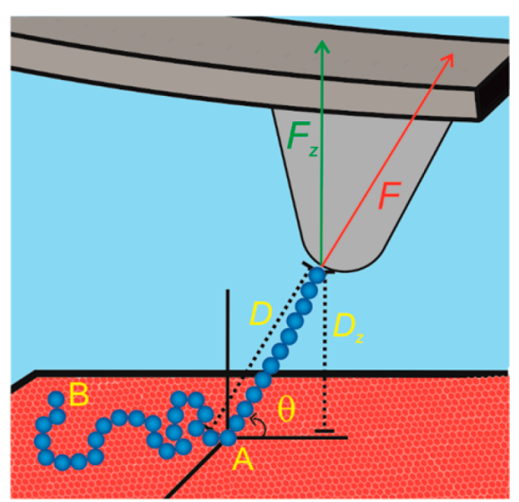

Figure 1. Schematic diagram of the experiment. The polymer is endgrafted to the AFM tip, adsorbed on the substrate first, and then pulled (under various angles) with respect to the surface. The desorption force $F$ is geometrically defined by the measured $F_{z}$ and the applied pulling angle $\theta . D$ and $D_{z}$ represent the desorption length and the measured length in the $\mathrm{z}$ direction, respectively. Angle-dependent measurements are performed by controlling the polar angle $\theta$, not considering the azimuthal angle $\varphi$. A, Last adhering segment in the scenario depicted; B, chain end.

Pulling in the Surface-Normal Direction. Typical experimental results for end-grafted PHEMA and PMMA are shown in Figure 2. In the first series of experiments, a series of approach-retraction force profiles was recorded as the polymer was pulled in the surface-normal direction. The force profiles varied from one approach-retraction cycle to another, and single molecule events were observed in approximately $20 \%$ of the force spectroscopy experiments for PHEMA and in less than $1 \%$ for PMMA. PMMA shows a low probability of events compared to PHEMA, which results from the hydrophobicity and tight precipitated coil conformation of the polymer chain in water and weak interaction with the substrates. The rest of the force curves show no single-polymer events at all (unspecific adhesion force signature could only be observed). When the polymer was bridging the tip and the substrate, one could distinguish two different types of events: the successive unbinding of individual segments, all bound to the surface in the so-called "train" conformation, until the last segment (chain end; B in Figure 1) becomes desorbed, and continuous increase in the restoring force with distance (i.e., polymer elongation). In most cases, the force profiles on retraction exhibited plateaus of constant force with increasing extension, as shown in Figure 2 (top left). Such desorption behavior has been observed and is well established in previous work. ${ }^{4,9,11}$ Here, the plateaus of constant force reflect the desorption of successive chain segments, whereas the complete desorption of the entire polymer chain results in a sudden drop of the adhesive force. The desorption force is independent of the chain length as would be expected for the "train" conformation of the molecule 
a
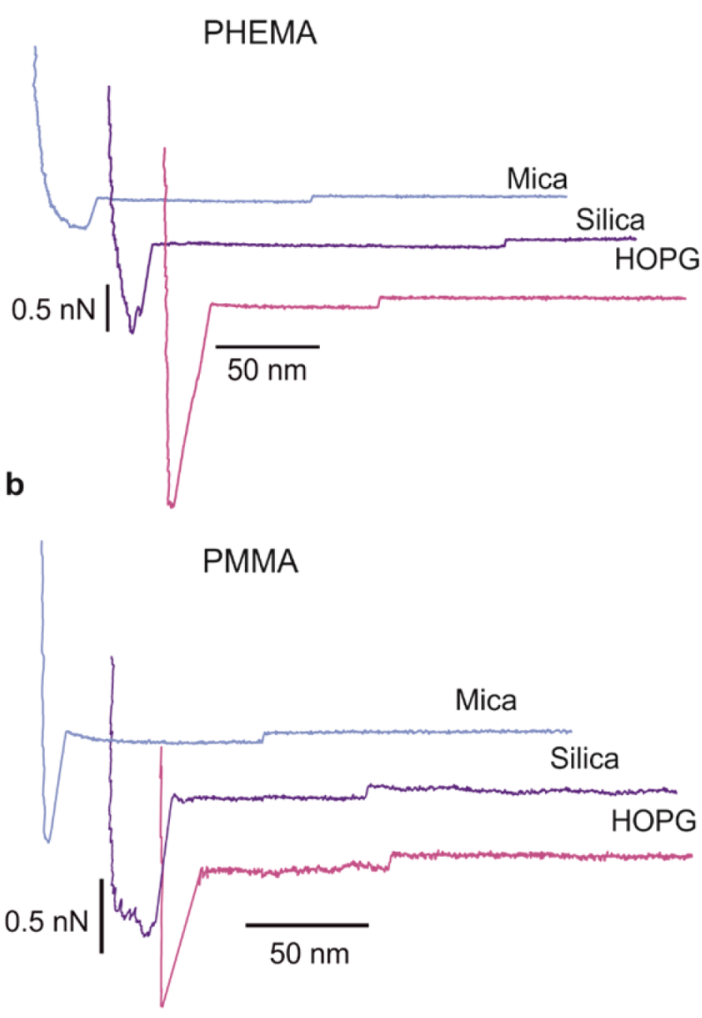

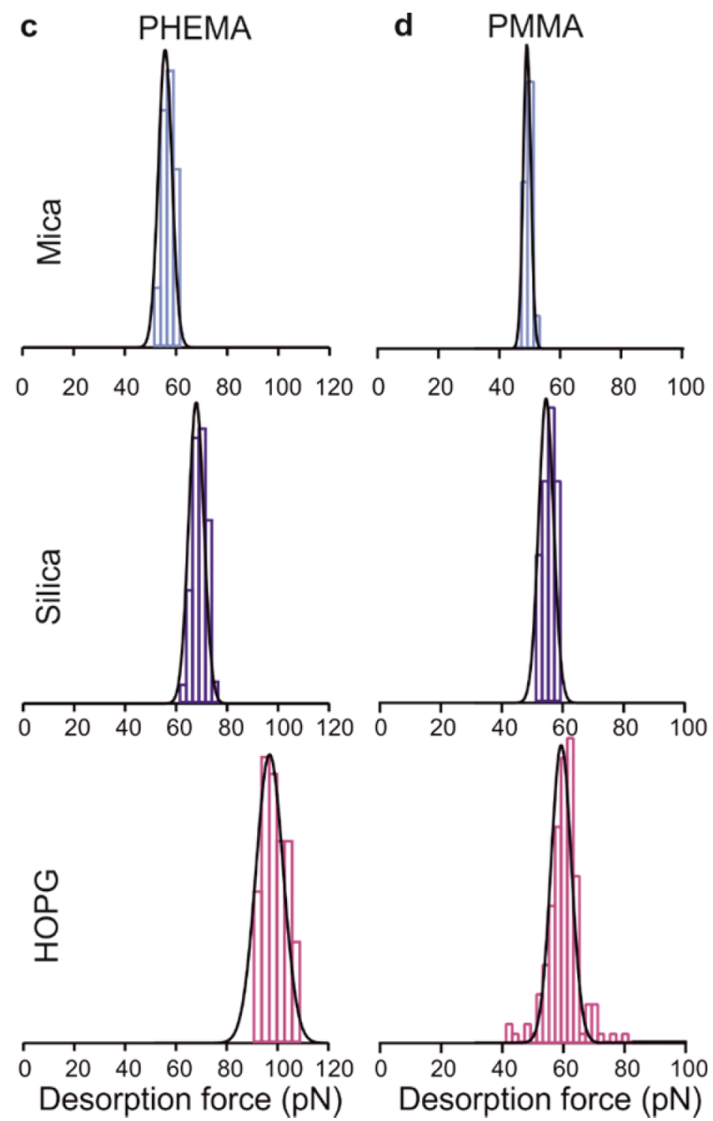

Figure 2. Desorption of end-grafted polymers from different substrates in ultrapure water. Typical retraction force curves for (a) PHEMA and (b) PMMA, as captured for the polymer desorbing in the surface-normal direction. Histograms of the desorption forces for (c) PHEMA and (d) PMMA recorded on mica, silica, and HOPG, from top to bottom. The vertical axes correspond to the number of counts, normalized to the maximum. The black lines show the Gaussian fit of the desorption forces.

on the surface. We confine ourselves in this analysis to "trainlike" adsorption.

The measurements are performed in thermodynamic quasiequilibrium, meaning that the bonds involved in the adsorption process dissociate and reassociate on a much faster time scale than the experimental desorption process. ${ }^{26}$ This assumption is supported experimentally by the finding that the desorption forces are independent of the bond loading rate used in the experiments, as we reported elsewhere. ${ }^{15}$ In the case of PHEMA, only a very small percentage of the total force events $(<0.2 \%)$ exhibited sharp sawtooth-like patterns in the force curves reflecting the stretching of sections of the individual polymer chains. In this case, the polymer-surface bonds were stable and relaxed slowly (the chain was pinned, and one could observe a nonlinear attractive peak in the retraction curve). The polymer stretching was much more likely for PMMA. We shall not address stretching of isolated single chains in this study; stretching of this type has been well established ${ }^{10,12,27,28}$ in the literature. Please note that because of the "unspecific" interaction of the silicon nitride tips with the substrate, force-extension curves occasionally show a high unspecific adhesion peak at short tip-substrate distances. However, in our measurements, the polymer chains exhibit sufficiently large contour lengths so that the unspecific adhesion peak does not affect the measured distributions of the desorption force. An example of the scatter plot of desorption force versus contour length for PHEMA on silica can be found in the Supporting Information (Figure S1).
The difference in the desorption forces of end-grafted PHEMA and PMMA caused by the interaction with various substrates was investigated on mica, silica, and HOPG. To this end, for each force curve obtained, we measured the magnitude of the average desorption force from individual plateaus. Histograms of the desorption forces in Figure 2c,d show that the distribution of the desorption forces is approximately Gaussian. (The fit parameters are shown in the Supporting Information.) The desorption force represents a direct measure of the interaction between the polymer and the surface in the solvent in question. The nature of the polymer and the surface substantially influences the magnitude of the desorption force, as can clearly be seen in Figure 2. The values of the average desorption forces and the corresponding standard deviations are summarized in Figure 3. The desorption force increases with increasing hydrophobicity of the substrate, i.e., with increasing adsorption strength. This trend is more pronounced for PHEMA and is very weak for PMMA. Depending on the substrate in question, the desorption forces lie in the range of $55-100 \mathrm{pN}$ for PHEMA and 48-60 pN for PMMA (Table 1). These findings compare well to the values reported for PHEMA previously ${ }^{29}$ and the desorption of other synthetic polymers or single stranded DNA. ${ }^{11,13,30-32}$ PHEMA and PMMA are neutral polymers composed of a hydrophobic backbone, having a hydrophilic ester group in their repeating unit, carrying a dipole due to the carbonyl group and featuring different side groups. The free hydroxyl groups of PHEMA make the polymer hydrophilic and lead to a relatively high 


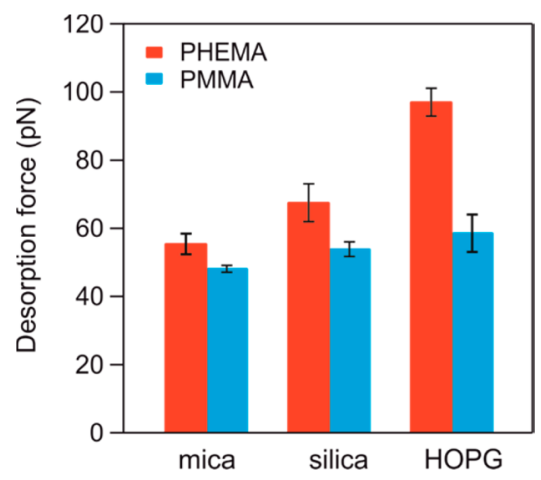

Figure 3. Desorption of end-grafted PHEMA and PMMA from different substrates in ultrapure water. Average desorption forces for PHEMA and PMMA are shown, recorded for mica, silica and HOPG with the corresponding standard deviations.

Table 1. Statistical Analysis of the Adhesion Data of PHEMA and PMMA on Various Surfaces with the Corresponding Standard Deviations

\begin{tabular}{|c|c|c|c|c|c|}
\hline \multirow[b]{2}{*}{ Surface } & \multirow[b]{2}{*}{$\begin{array}{l}\text { Water Contact } \\
\text { Angle (deg) }\end{array}$} & \multicolumn{2}{|c|}{ Desorption Force $(\mathrm{pN})$} & \multicolumn{2}{|c|}{$\begin{array}{c}\text { Adsorption } \\
\text { Energy per } \\
\text { Monomer }(k T)\end{array}$} \\
\hline & & PHEMA & PMMA & PHEMA & PMMA \\
\hline Mica & $\leq 5$ & $55.7 \pm 3.0$ & $48.4 \pm 1.0$ & 3.7 & 3.2 \\
\hline Silica & $18 \pm 5$ & $67.8 \pm 5.5$ & $54.2 \pm 2.1$ & 4.5 & 3.6 \\
\hline HOPG & $71 \pm 5$ & $97.3 \pm 7.0$ & $58.9 \pm 5.4$ & 6.4 & 3.9 \\
\hline
\end{tabular}

degree of hydration. ${ }^{33}$ The side groups of PMMA are composed of only short hydrophobic methyl groups that make the chain hydrophobic.

The PHEMA chains swell in aqueous solution with a hydration layer formed via hydrogen bonding with water molecules. ${ }^{34}$ The strong increasing trend observed for PHEMA adhesion can be explained by hydrophobic interactions and a high van der Waals attractive interaction between the hydrophobic backbone of the polymer and the substrate. An adsorption energy per monomer ranging from 3.7 to $6.7 k T$ is observed, where $k T$ refers to the thermal energy (see Table 1). Similar adsorption energies have been reported for synthetic polymers and polyelectrolytes. ${ }^{35}$ Lower adsorption energies obtained for more hydrophilic surfaces can be explained by the adsorption resistance at the hydrophilic surface that results from strongly bound interfacial water due to water-surface hydrogen bonding, suppressing direct polymer-surface interactions. ${ }^{4}$ PMMA exhibits only a weak variation in the adsorption energy on different substrates in aqueous solution. Unlike PHEMA, water is a nonsolvent for PMMA, so the surface-tethered chains are in a collapsed globule state, excluding the solvent. ${ }^{36}$ In water, the PMMA chains form self-assembled domains in a pearl-necklace conformation. ${ }^{37} \mathrm{We}$ observed an increased adhesion on more hydrophobic substrates, i.e., HOPG, with a high frequency of stretching events that can be rationalized by interaction forces of a hydrophobic character.

Adhesion energies obtained experimentally were compared to the theoretical values for the polymer interacting with various substrates across a medium using the Lifshitz theory. ${ }^{38}$ The calculation procedure is described in the Supporting Information. The trend and variation of the magnitude of the calculated values of adhesion energies for various substrates (as presented in Table 2) corroborate the measured adsorption energies per segment (Table 1), i.e., the adhesion increases in

Table 2. Theoretical Values for Macroscopic Phases 1 and 2 Interacting Across a Medium 3

\begin{tabular}{lcccc} 
Phase & $\begin{array}{c}\text { Dielectric } \\
\text { Constant } \\
\varepsilon^{38,39}\end{array}$ & $\begin{array}{c}\text { Refractive } \\
\text { Index } \\
n^{38,40,41}\end{array}$ & $\begin{array}{c}\text { Hamaker } \\
\text { Constant } \\
\left(10^{-20} \mathrm{~J}\right)\end{array}$ & $\begin{array}{c}\text { Adhesion } \\
A_{132} \\
\text { Energy W } \mathbf{W}^{38} \\
(k T)\end{array}$ \\
Mica & 4 & 1.56 & 0.9 & 1.5 \\
Silica & 11 & 1.45 & 1.6 & 2.6 \\
HOPG & 10000 & 1.5 & 2.8 & 4.5 \\
Water & 80 & 1.33 & & \\
Polymer & 2.6 & 1.49 & & \\
\hline
\end{tabular}

the order of mica-silica-HOPG. The absolute values differ as the calculations neglect contributions from other short-range forces.

In the desorption experiments, we occasionally observed plateaus of constant force with several steps in the force profiles. Such "stapled" plateaus can reflect desorption involving loops within individual polymer molecules or contributions from several polymer chains pulled off the substrate. In such cases, molecules of different lengths are simultaneously adsorbed on the surface, and the steps represent their desorption, one after the other, until the last polymer is fully detached. The last step then reflects the desorption of a single polymer from the substrate (see Figure S2 Supporting Information). The probability of multiple steps in the desorption force varied with the substrate. The steps were observed in $1.2 \%$ of all cases for mica, in $7 \%$ for silica, and in $88.1 \%$ for HOPG. This trend can be rationalized by different polymer-surface interactions and possible loop formation with increased hydrophobicity of the substrate.

Angle-Dependent Pulling. We investigated angle-dependent adhesion of tip end-grafted PHEMA while desorbed from various substrates at different pulling angles in ultrapure water by SMFS. We aimed to measure the magnitude and control the direction of forces acting on the polymers using a customwritten software, in combination with a commercial AFM instrument, as reported in detail elsewhere. ${ }^{15}$ The experimental strategy is illustrated in Figure 1. The AFM tip with end-grafted polymer approached the surface, the polymer chain was adsorbed on the surface and subsequently pulled away at different angles with respect to the surface. In our experiments, we only varied polar angles, as the chain orientation on the surface was not known, and the variation in the azimuthal angles could therefore not be controlled and is not expected to provide meaningful information. Furthermore, by considering the FJC model, ${ }^{42}$ the desorption of the polymer segment by segment with a constant force suggests that only the orientation of two neighboring points at the time is relevant, and the remaining segments of the polymer chain are attached to the surface. During the experiments, the force measured through the deflection of the AFM cantilever, $F_{z}$, represents the vertical component of the force applied to the polymer molecule, $F$. The desorption force acting along the contour of the polymer was then determined from the measured $F_{z}$ (where $F_{z}=F$ sin $\theta$ ) using the pulling geometry as given in Figure 1. Similarly, the measured extension, $D_{z}$, is only the projection of the distance between the two anchor points.

The other components of the force vector (e.g., lateral) can cause deflection of the AFM cantilever (twisting the cantilever). Several mechanisms will contribute to the measured desorption 

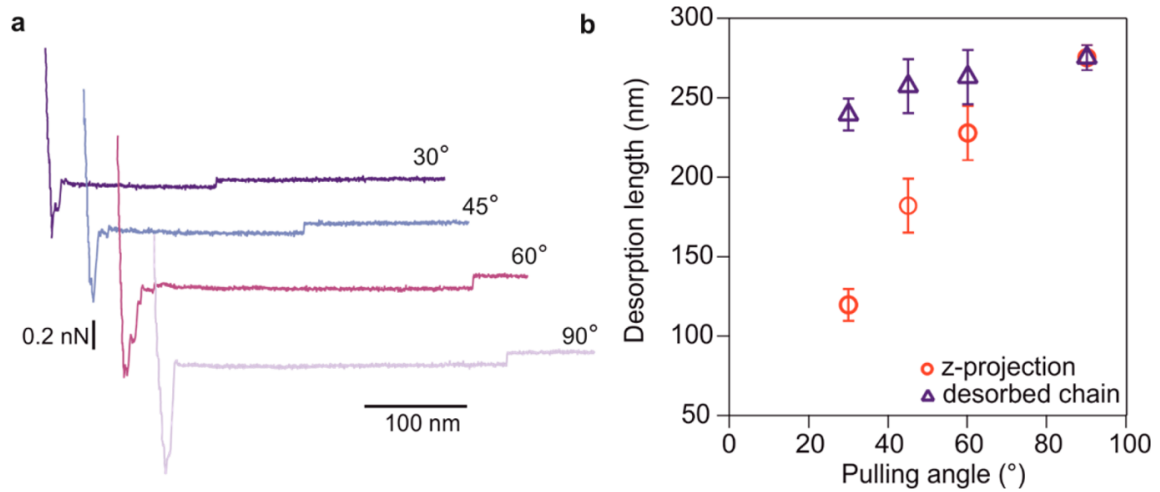

Figure 4. Angle-dependent desorption of end-grafted PHEMA from silica in ultrapure water. (a) Force profiles upon retraction exhibiting plateaus of constant force observed for pulling angles in the range between $30^{\circ}$ amd $90^{\circ}$. (b) Average desorption length plotted as a function of pulling angle with corresponding standard deviations.

force: adhesion, friction, and elasticity of the polymer as well. We have recorded the lateral deflection signal during the angledependent experiments to verify if there is a measurable lateral torsion of the cantilever. The lateral deflection signal did not display any detectable change while desorbing a polymer chain at various angles. ${ }^{15}$

Figure 4a shows typical force profiles of PHEMA obtained in angle-dependent adhesion measurements upon retraction at various pulling angles on silica in ultrapure water. The pulling angles were varied in the range between $30^{\circ}$ and $90^{\circ}$. The $\mathrm{z}-$ component of the desorption length decreases with a decreasing pulling angle as the polymer is continuously desorbed from the surface (Figure $4 \mathrm{~b}$ ). These findings correlate well with the geometrical prediction of the experiment. While desorbing a polymer chain, we consider a mechanism where a polymer chain can be peeled from the surface without changing the binding sites of the monomers. Within the FJC model we assume that only two neighboring connected segments are relevant for polymer pulling. However, due to variable surface conformations and the change of force attack at shallow angles, an error between contour length and experimentally measured length arises (Figure $4 \mathrm{~b}$ ). If we pull a chain perpendicular to the substrate and if no displacement has taken place, then the desorption (contour) length $Z$ can be estimated as $Z=\sqrt{L^{2}-R_{\mathrm{ee}}{ }^{2}}$, where $L$ is contour length and $R_{\mathrm{ee}}$ end-toend distance. The deviation between contour length and pulling length would be approximately $\approx 1.5 \%$. We neglect this error and assume that the pull length ( $Z$ travel) for perpendicular pull is the same as the contour length. Figure $4 \mathrm{~b}$ shows that the deviation is most noticeable for the shallowest pulling angle. We have estimated the deviation between contour length and experimentally measured length for $30^{\circ}$ and $90^{\circ}$ pull to be approximately $15 \%$, taking into account similar polymer chains ${ }^{43}$ imaged on the same substrate (estimated deviation here 13\%), end-to-end distance, contour length and experimental geometry. Details about the error estimation are provided in the Supporting Information.

We state that over the pulling experimental time scales chain lateral diffusion can be neglected. We base this statement on the fact that single chains can be visualized in a given conformation at substrates by tapping mode imaging ${ }^{13,43}$ over a time scale of several minutes. A single pulling measurement in our case takes typically 4 s. Obviously, if nonmoving chains can be imaged over several minutes, then we can assume that lateral diffusion would be negligible within the time scale of our experiment. We can also rule out the option that the XY offset of the actually desorbing segment closely follows the tip motion, i.e., in this case the pulling length would be equal to the contour length. This cannot be justified by the data (Figure 4b). Qualitatively, the same findings have been observed for the angle-dependent desorption of PHEMA from other substrates, as well. Thus, we are left with two options: (a) no slide, (b) some slide but variation at XY offset closely following the tip motion is not fulfilled.

The average desorption forces of end-grafted PHEMA as a function of the pulling angle measured on various substrates in ultrapure water are summarized in Figure 5. All surfaces exhibit the same behavior; the desorption force increases monotonically as the pulling angle decreases. The distribution of the average desorption forces for the individual pulling angles was
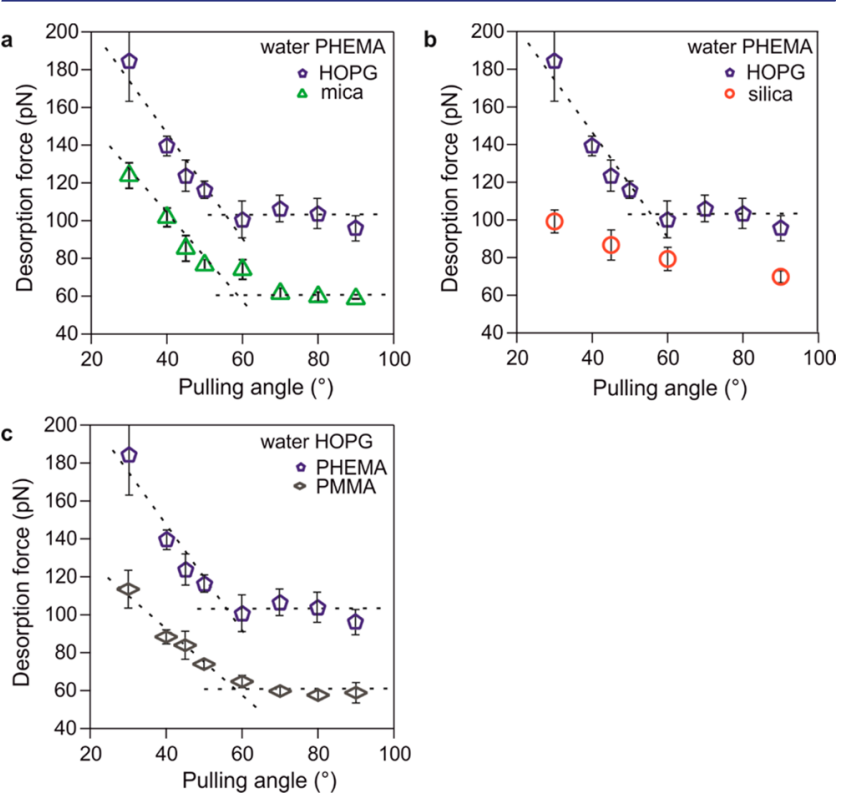

Figure 5. Angle-dependent adhesion of various systems. The average desorption forces with corresponding standard deviations recorded for different pulling angles on HOPG in ultrapure water compared to (a) end-grafted PHEMA desorbed from mica, (b) end-grafted PHEMA desorbed from silica, and (c) end-grafted PHEMA and PMMA. The dashed lines serve only to guide the eye. The desorption force acting along the contour of the polymer $F$ was then determined from $F_{z}$ (where $F_{z}=F \sin \theta$ ) using the pulling geometry as given in Figure 1 . 
relatively narrow and approximately Gaussian (example shown in Supporting Information for HOPG, Figure S3). Desorption measurements at a particular pulling angle represent a statistical result arising from many possible chain conformations on the substrate but our finding supports the assumption that the orientation of the chain on the surface (i.e., variation in the azimuthal angle) has a negligible effect on the polymer desorption on homogeneous, unstructured substrates. We note that the change in the azimuthal angle might play a significant role on polymer desorption from heterogeneous, structured ("stripped") or anisotropic substrates. The importance of pulling angle for such surfaces has been demonstrated theoretically. ${ }^{22}$

The magnitude of the desorption force depends on the strength of the polymer-surface interactions in question; for all surfaces, decreasing the pulling angle resulted in enhanced adhesion of the polymer chain, as predicted theoretically. ${ }^{18,21,24}$ As shown in Figure 5a, we display in detail the angle-dependent desorption of PHEMA on two substrates, namely, on mica and HOPG. The average desorption force is approximately constant at higher pulling angles, but by decreasing the angle beyond $50^{\circ}$, the desorption force abruptly increases. This we interpret as the observation of the fingerprints of the theoretically predicted adsorption-desorption transition with varying pulling angles. The theoretical predictions ${ }^{19,21}$ rely on partially directed walk models in three dimensions of a polymer tethered at one end to a surface and being pulled at fixed angles away from the point of tethering. At a given temperature, pulling angle variations lead to changes in the interaction force. The transition angle would not change when the interaction energy is varied; however, the temperature dependence of the force magnitude would vary. The lattice model for polymer adhesion considered by Orlandini and Whittington, ${ }^{19}$ and by Osborn and Prellberg, ${ }^{21}$ predicts that at first, for pulling directions close to normal to the surface, there is only a small dependence on pulling angle. However, the dependence becomes more marked as the angle decreases. At very large forces (and so at very small angles where the required force is large), there is a problem with the model. One would expect that the walk would eventually follow the pulling direction but this is not entirely possible for a lattice model. This means that the model overestimates the force required for desorption at small angles. This only becomes a major issue at angles less than about $30^{\circ}$ and, at larger angles, there is very good qualitative agreement between theory and experiment. As Figure 5a,b shows, the transition angle does not change with the variation of the substrate; only the magnitude of the measured force varies as the result of the difference in the interaction energy of PHEMA with the various substrates.

Figure 5c shows the average desorption force vs pulling angle for PHEMA and PMMA. For the two polymers, we observed qualitatively the same trend, i.e., an enhancement of the polymer adhesion with decreasing pulling angle. The magnitude of the force varies because of different polymersurface interactions.

Figure 6 offers a semiquantitative comparison between the theory and the experiments. The shape of the force dependence on the pulling angle is the same, with only a scaling factor difference. The theory is based on a partially directed walk on a lattice and there is no "chemistry" in the model, i.e., all surfaces and all polymers are treated as being identical, as described in the Introduction. However, it is interesting to note that the experimental data all more or less collapsed to a single curve

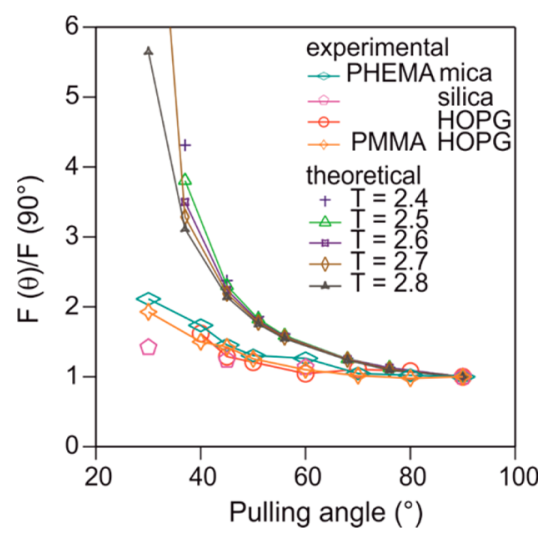

Figure 6. Desorption force at pulling angle $\theta$ normalized by force at $90^{\circ}$ as a function of the pulling angle. Experimental data obtained for the desorption of PHEMA and PMMA on various substrates are compared to theoretical critical force curves versus pulling angle for given range of temperatures as based on partially directed walk models of adsorbed polymers. ${ }^{19}$

when normalized by $F\left(90^{\circ}\right)$. This suggests that the angular term is largely independent of the system, showing a universal behavior. Theoretical force values were determined from the critical force-temperature curves for various values of the angle at which the force is applied. We have compared values obtained for the temperature range of 2.4-2.8, i.e., below the critical adsorption temperature of about 3.03 (the temperature at which desorption occurs without a force). ${ }^{21}$ At a particular temperature $T$, the force required for the polymer desorption changes, depending strongly on $T$ at each angle. However, the ratio of $F(\theta) / F\left(90^{\circ}\right)$ does not depend much on the temperature. Dividing by the force at $90^{\circ}$ value significantly removes the temperature dependence. (We note that all our studies were performed at isothermal conditions at room temperature.)

\section{CONCLUSION}

In conclusion, we addressed the single polymer chain response to external force applied at various angles in aqueous solution. The experiments performed with different polymers (PHEMA and PMMA) and on various substrates demonstrated an enhancement of the polymer adhesion while decreasing the pulling angle, in full agreement with theoretical predictions. 19,21 A clear trend for the adsorption-desorption transition angle was found experimentally by performing direction-dependent force spectroscopy experiments. The dependence of the desorption force on the pulling angle significantly increased at smaller angles and showed with decreasing angle the adsorption-desorption transition, in qualitative agreement with theoretical predictions. ${ }^{19,21}$ The angular dependence of the normalized desorption force exhibits a universal behavior. A semiquantitative comparison between the theory and the experiments has been offered. The force dependence on the pulling angle shows the same shape, with only a scaling factor difference.

\section{EXPERIMENTAL SECTION}

Materials. 3-Aminopropyl trimethoxysilane (APTMS, 97\%), triethylamine (TEA, 99.5\%), copper(II) bromide $\left(\mathrm{CuBr}_{2}, 99 \%\right)$, 2,2'-bipyridine (bipy, 98\%), 2-bromoisobutyryl bromide (BIBB, 98\%), 1-bromocarbonyl-1-methylethyl acetate (BMA, 96\%), $N, N, N^{\prime}, N^{\prime}, N^{\prime}$-pentamethyldiethylenetriamine (PMDETA, 98\%), and 
L-ascorbic acid (LAA) were purchased from Sigma-Aldrich and were used as received. Toluene (AR) and ethanol (absolute) were purchased from Biosolve and Merck, respectively. All water used in the experiments was ultrapure (Millipore Milli- $Q$ grade). 2Hydroxyethyl methacrylate (HEMA, 97\%) and methyl methacrylate (MMA, 99\%) were purchased from Sigma-Aldrich and purified from inhibitors by passing through a basic alumina column. Copper(I) chloride ( $\mathrm{CuCl}, 98 \%)$ was purchased from Sigma-Aldrich, purified by stirring in glacial acetic acid for several hours, filtered, washed with absolute ethanol, and vacuum-dried. The native oxidized silicon wafer surfaces were purchased from Okmetic (Vantaa, Finland). The silicon cantilevers were obtained from Nanosensors (Neuchâtel, Switzerland).

Synthesis of Polymer Chains by SI-ATRP. The synthesis of the PHEMA and PMMA polymer chains was achieved through classical ATRP and activators regenerated by electron transfer (ARGET) ATRP, respectively. Polymer brushes and single polymer chains were end-grafted from reference substrates of planar silicon and AFM tip, respectively. The SI-ATRP of PHEMA and PMMA on silicon surfaces and AFM tips was performed simultaneously in the same batch. Each polymerization step was verified on the planar substrates. Briefly, the synthesis consisted of four steps: the pretreatment/cleaning of the surfaces, the amino functionalization via silanization of the substrates, the coupling with active and inactive ATRP-initiators via an acylation reaction and the controlled radical polymerization (CRP) of the monomers. The native oxidized silicon wafer surfaces were cleaned with piranha solution, and the cantilevers were plasma cleaned with an SPI Plasma Prep II instrument (SPI Supplies, West Chester, USA) in an oxygen-enriched atmosphere prior to use. Subsequently, the planar reference surfaces and cantilevers were silanized for $12 \mathrm{~h}$ in the gas phase in an evacuated container in the presence of APTMS and washed with toluene and ethanol. To control the polymer grafting density, the cantilevers with amino-functionalities were coupled with active and inactive ATRP initiator precursors: BIBB and 1bromocarbonyl-1-methylethyl acetate (BMA), respectively. The cantilevers were immersed in toluene with TEA, to which BIBB and BMA were added in the desired molar ratio. The surfaces were thoroughly washed with toluene, ethanol, and water and dried under the stream of nitrogen. PHEMA polymer chains were synthesized via SI-ATRP, while the synthesis of PMMA polymer chains was achieved via SIARGET-ATRP. The PHEMA and the PMMA-modified samples were washed with water then ethanol and dried in a stream of nitrogen. Further details on the preparation of the surfaces can be found in the Supporting Information.

Film Characterization. Surface functionalization and the resulting polymer films of the reference planar silicon substrates were characterized by FTIR spectroscopy, contact angle measurements, and AFM imaging to ensure that each step of the polymerization of HEMA and MMA on the AFM cantilever was successful. FTIR spectra were obtained using a Vertex 70v spectrometer (Bruker) with a nitrogen-cooled cryogenic detector. Background spectra were obtained using a cleaned silicon oxide substrate. Contact angle measurements were performed with an optical contact angle device (OCA 15, Dataphysics, Germany) equipped with an electronic syringe unit and connected to a charge-coupled device (CCD) camera. Values of the static contact angles of ultrapure water were measured using the sessile drop mode. The dry thickness of the polymer brush on the planar substrate was obtained by measuring the step height of scratches gently made on the silicon. A Dimension D3100 AFM equipped with a hybrid scanner and a NanoScope IVa controller (Digital Instruments, Veeco-Bruker, Santa Barbara, CA) were employed, operated in intermittent-contact mode in air using cantilevers (Nanoworld, Neuchâtel, Switzerland) with a force constant of approximately 42 $\mathrm{N} / \mathrm{m}$ and a resonance frequency of $320 \mathrm{kHz}$ at a scan rate of $1 \mathrm{~Hz}$.

Angle-Dependent SFMS. SMFS measurements were carried out in ultrapure water (Millipore Milli-Q grade, $\mathrm{pH} 7$ ) in contact mode with a commercial AFM instrument Molecular Force Probe 3D (MFP3D) (Asylum Research, Santa Barbara, CA). The cantilevers (PointProbe Plus Contact Mode, Nanosensors, Neuchâtel, Switzerland) with the end-grafted polymer chain, prepared as described above, had a nominal tip radius smaller than $7 \mathrm{~nm}$ and a resonance frequency of $10-15 \mathrm{kHz}$ in water. The SMFS experiments were performed with different polymers (PHEMA and PMMA) on various substrates, namely, mica, silica, and highly ordered pyrolytic graphite (HOPG). High-grade mica and HOPG were obtained from Plano (Wetzlar, Germany) and cleaved with adhesive tape in air prior to use. The native oxidized silicon wafer surfaces were plasma cleaned with an SPI Plasma Prep II instrument (SPI Supplies, West Chester, USA) in an oxygen-enriched atmosphere for $20 \mathrm{~min}$ prior to use.

In SFMS, a series of several thousand approach-retraction force curves was recorded with a sampling rate of $2 \mathrm{kHz}$, whereby the deflection of the cantilever and the vertical piezo displacement were acquired as a function of time. After the surface was contacted, the AFM tip with end-grafted polymer was retracted under different pulling angles relative to the substrate, as shown in Figure 1. The pulling angle control was programmed based on Igor Pro and Asylums Research's built-in functions. In detail, the tip was retracted perpendicularly to the surface with a constant velocity while the substrate was simultaneously moved in the $\mathrm{x}$ and $\mathrm{y}$ directions. By precisely controlling the velocities in the Cartesian directions, the desired pulling angle was set. The experimental strategy for the directional force experiments is addressed in more detail elsewhere. ${ }^{15}$ To preserve the functionalized tip, the maximum applied force upon contact with the surface was kept smaller than $4 \mathrm{nN}$. The cantilever spring constants were in the range of $0.12-0.28 \mathrm{~N} / \mathrm{m}$ as measured by thermal fluctuations in air. ${ }^{44}$ The retraction velocity used was $250 \mathrm{~nm} /$ s. Retraction force curves were very similar when the velocity was varied in the range of 25-18 $000 \mathrm{~nm} / \mathrm{s}$, and the recorded desorption force was the same for all velocities within the experimental error (data not shown). Changing the dwell time toward the surface did not have any effect on the recorded force response. All AFM experiments were carried out at room temperature of $20 \pm 1{ }^{\circ} \mathrm{C}$. The resulting single molecule force profiles were analyzed with the program Igor Pro (Wavemetrics) with custom procedures. Plateau force and length were extracted for each force curve. The values of each measurement were plotted in a histogram and fitted with Gaussian distributions. The maximum of the Gaussian functions was extracted and the standard deviation was plotted as error.

\section{ASSOCIATED CONTENT}

\section{S Supporting Information}

The Supporting Information is available free of charge on the ACS Publications website at DOI: 10.1021 /jacs.8b02851.

Detailed synthetic procedure for the preparation of polymer chains via ATRP; scatter plot of the desorption force versus contour length for PHEMA desorbing from silica substrate; example illustrating multiple steps in the force extension curve of PHEMA desorbing from silica substrate; histograms of desorption force for different pulling angles on HOPG substrate; calculation of the adhesion energies; estimation of error between chain contour length and experimental pull length; Gaussian fit parameters for desorption of end-grafted polymers from different substrates in ultrapure water (PDF)

\section{AUTHOR INFORMATION}

\section{Corresponding Author}

*g.j.vancso@utwente.nl

ORCID

Julius G. Vancso: 0000-0003-4718-0507

\section{Notes}

The authors declare no competing financial interest.

\section{ACKNOWLEDGMENTS}

This research was supported by the Swiss National Science Foundation (SNSF) (L.G. thanks SNSF for the financial 
support via Fellowship for Prospective Researchers Grant No. P2GEP2_161783), MESA+ and University of Twente. The authors thank M. Cirelli for the help with the polymer synthesis by ATRP.

\section{REFERENCES}

(1) Duarte, A. P.; Coelho, J. F.; Bordado, J. C.; Cidade, M. T.; Gil, M. H. Prog. Polym. Sci. 2012, 37, 1031.

(2) Zhu, X.; Guo, S.; Jańczewski, D.; Parra Velandia, F. J.; Teo, S. L.M.; Vancso, G. J. Langmuir 2014, 30, 288.

(3) Adamczyk, Z.; Barbasz, J.; Ciesla, M. Langmuir 2011, 27, 6868.

(4) Schwierz, N.; Horinek, D.; Liese, S.; Pirzer, T.; Balzer, B. N.; Hugel, T.; Netz, R. R. J. Am. Chem. Soc. 2012, 134, 19628.

(5) Borkner, C. B.; Elsner, M. B.; Scheibel, T. ACS Appl. Mater. Interfaces 2014, 6, 15611.

(6) Kumar, A.; Srivastava, A.; Galaev, I. Y.; Mattiasson, B. Prog. Polym. Sci. 2007, 32, 1205.

(7) Balzer, B. N.; Gallei, M.; Hauf, M. V.; Stallhofer, M.; Wiegleb, L.; Holleitner, A.; Rehahn, M.; Hugel, T. Angew. Chem., Int. Ed. 2013, 52, 6541.

(8) Rose, S.; Prevoteau, A.; Elziere, P.; Hourdet, D.; Marcellan, A.; Leibler, L. Nature 2014, 505, 382.

(9) Friedsam, C.; Seitz, M.; Gaub, H. E. J. Phys.: Condens. Matter 2004, 16, S2369.

(10) Rief, M.; Gautel, M.; Oesterhelt, F.; Fernandez, J. M.; Gaub, H. E. Science 1997, 276, 1109.

(11) Hugel, T.; Grosholz, M.; Clausen-Schaumann, H.; Pfau, A.; Gaub, H.; Seitz, M. Macromolecules 2001, 34, 1039.

(12) Oesterhelt, F.; Rief, M.; Gaub, H. E. New J. Phys. 1999, 1, 6.

(13) Grebikova, L.; Maroni, P.; Muresan, L.; Zhang, B. Z.; Schlüter,

D. A.; Borkovec, M. Macromolecules 2013, 46, 3603.

(14) Haschke, H.; Miles, M. J.; Koutsos, V. Macromolecules 2004, 37, 3799.

(15) Grebíková, L.; Gojzewski, H.; Kieviet, B.; Klein Gunnewiek, M.; Vancso, G. Rev. Sci. Instrum. 2017, 88, 033705.

(16) Kühner, F.; Erdmann, M.; Gaub, H. E. Phys. Rev. Lett. 2006, 97, 218301.

(17) Ke, C.; Jiang, Y.; Rivera, M.; Clark, R. L.; Marszalek, P. E. Biophys. J. 2007, 92, L76.

(18) Iliev, G. K.; Whittington, S. G. J. Phys. A: Math. Theor. 2012, 45, 185003.

(19) Orlandini, E.; Whittington, S. G. J. Phys. A: Math. Theor. 2010, 43,485005

(20) Serr, A.; Netz, R. R. Europhys. Lett. 2006, 73, 292.

(21) Osborn, J.-a.; Prellberg, T. J. Stat. Mech.: Theory Exp. 2010, 2010, P09018.

(22) Iliev, G.; Whittington, S. G. Phys. Rev. E 2013, 88, 052105.

(23) Kuhner, F.; Erdmann, M.; Sonnenberg, L.; Serr, A.; Morfill, J.;

Gaub, H. E. Langmuir 2006, 22, 11180.

(24) Serr, A.; Netz, R. R. Europhys. Lett. 2007, 78, 68006.

(25) Matyjaszewski, K. Macromolecules 2012, 45, 4015.

(26) Friedsam, C.; Gaub, H. E.; Netz, R. R. Biointerphases 2006, 1, MR1.

(27) Giannotti, M. I.; Vancso, G. J. ChemPhysChem 2007, 8, 2290.

(28) Liu, N.; Zhang, W. ChemPhysChem 2012, 13, 2238.

(29) Zhang, D.; Ortiz, C. Macromolecules 2005, 38, 2535.

(30) Friedsam, C.; Becares, A. D.; Jonas, U.; Gaub, H. F.; Seitz, M. ChemPhysChem 2004, 5, 388.

(31) Kienle, S.; Gallei, M.; Yu, H.; Zhang, B. Z.; Krysiak, S.; Balzer, B. N.; Rehahn, M.; Schluter, A. D.; Hugel, T. Langmuir 2014, 30, 4351.

(32) Manohar, S.; Mantz, A. R.; Bancroft, K. E.; Hui, C.-Y.; Jagota, A.; Vezenov, D. V. Nano Lett. 2008, 8, 4365.

(33) Zhang, D.; Ortiz, C. Macromolecules 2004, 37, 4271.

(34) Desseaux, S.; Hinestrosa, J. P.; Schüwer, N.; Lokitz, B. S.; Ankner, J. F.; Kilbey, S. M.; Voitchovsky, K.; Klok, H.-A. Macromolecules 2016, 49, 4609.

(35) Friedsam, C.; Gaub, H.; Netz, R. Europhys. Lett. 2005, 72, 844.
(36) Flory, P. J. Principles of polymer chemistry; Cornell University Press: Ithaca, 1953.

(37) Gunari, N.; Walker, G. C. Langmuir 2008, 24, 5197.

(38) Israelachvili, J. N. Intermolecular and surface forces, 3rd ed.; Academic Press: New York, 2011.

(39) Mazur, K. J. Phys. D: Appl. Phys. 1997, 30, 1383.

(40) Malitson, I. J. Opt. Soc. Am. 1965, 55, 1205.

(41) Beadie, G.; Brindza, M.; Flynn, R. A.; Rosenberg, A.; Shirk, J. S. Appl. Opt. 2015, 54, F139.

(42) Flory, P. Statistical mechanics of chain molecules; Hanser Publishers: Munich, 1969.

(43) Kumaki, J.; Nishikawa, Y.; Hashimoto, T. J. Am. Chem. Soc. 1996, 118, 3321.

(44) Proksch, R.; Schäffer, T. E.; Cleveland, J. P.; Callahan, R. C.; Viani, M. B. Nanotechnology 2004, 15, 1344. 